\title{
A New Drosophila POU Gene, pdm3, Acts in Odor Receptor Expression and Axon Targeting of Olfactory Neurons
}

\author{
Andrea L. Tichy, Anandasankar Ray, and John R. Carlson \\ Department of Molecular, Cellular, and Developmental Biology, Yale University, New Haven, Connecticut 06520-8103
}

\begin{abstract}
Olfaction depends on the differential activation of olfactory receptor neurons (ORNs) and on the proper transmission of their activities to the brain. ORNs select individual receptors to express, and they send axons to particular targets in the brain. Little is known about the molecular mechanisms underlying either process. We have identified a new Drosophila POU gene, pdm3, that is expressed in ORNs. Genetic analysis shows that $p d m 3$ is required for odor response in one class of ORNs. We find that $p d m 3$ acts in odor receptor expression in this class, and that the odor response can be rescued by the receptor. Another POU gene, acj6, is required for receptor expression in the same class, and we find a genetic interaction between the two POU genes. The results support a role for a POU gene code in receptor gene choice. $p d m 3$ is also expressed in other ORN classes in which it is not required for receptor expression. For two of these classes, $p d m 3$ is required for normal axon targeting. Thus, this mutational analysis, the first for a POU class VI gene, demonstrates a role for $p d m 3$ in both of the processes that define the functional organization of ORNs in the olfactory system.
\end{abstract}

Key words: POU gene; odor receptor; axon targeting; Drosophila; maxillary palp; antenna

\section{Introduction}

Each olfactory receptor neuron (ORN) makes two remarkable choices that underlie the sense of smell. ORNs select individual odor receptor genes to express, a process that determines which odors they detect, and they send axons to particular targets in the brain, which determines what behaviors are elicited by the odors. The molecular mechanisms underlying both choices are largely unknown.

ORNs in Drosophila are contained within two organs, the antenna and the maxillary palp. The odor response spectrum of an ORN class is conferred by the expression of one or a small number of odor receptor (Or) genes (Dobritsa et al., 2003; Hallem et al., 2004; Goldman et al., 2005). The organization of ORN classes is stereotyped, and depends on the proper selection of individual Or genes from among a large repertoire. ORNs send axons to individual glomeruli, which are spheroidal modules in the antennal lobe of the brain. ORNs that express the same receptor converge on the same glomerulus (Gao et al., 2000; Vosshall et al., 2000).

In Drosophila, the expression of particular Or genes, and hence the odor specificity of the ORN, has recently been found to depend on a regulatory code of cis-acting elements (Ray et al., $2007,2008)$. Transcription factors that act in the process of receptor gene expression include abnormal chemosensory jump 6

\section{Received June 11, 2008; accepted May 23, 2008.}

This work was supported by a National Institutes of Health (NIH) Training Grant 2T32GM007499-31, by a National Research Service Award to A.L.T., and by NIH grants to J.R.C. We thank Lei Bai and Tong-Wey Koh for critical reading of this manuscript.

Correspondence should be addressed to John R. Carlson, Department of Molecular, Cellular, and Developmental Biology, Yale University, New Haven, CT 06520-8103. E-mail: john.carlson@yale.edu.

A. Ray's present address: Department of Entomology, University of California, Riverside, CA 92521.

DOI:10.1523/JNEUROSCI.2063-08.2008

Copyright $\odot 2008$ Society for Neuroscience $\quad$ 0270-6474/08/287121-09\$15.00/0
(Acj6), a POU transcription factor. The acj6 gene was identified by a defect in olfactory behavior (McKenna et al., 1989; Ayer and Carlson, 1991; Clyne et al., 1999b). In a null mutant of acj6, some maxillary palp ORNs respond normally to odors, some are present but have lost response to all odors, and some undergo changes in odor specificity (Clyne et al., 1999b). Correspondingly, acj6 is required for the expression of a subset of Or genes (Clyne et al., 1999a).

In the present study, we identify a new POU gene, $p d m 3$, which is a POU gene of class VI. We find that $p d m 3$ is expressed in ORNs. In the first study of a POU VI mutant in any organism, we find that loss of $p d m 3$ results in loss of odor response in a class of ORNs in the maxillary palp. Correspondingly, the defective ORNs lose Or gene expression. The phenotypes can be rescued with a cDNA representing either $p d m 3$ or the affected Or gene, indicating a surprising degree of specificity to the odor-sensitivity phenotype. We demonstrate a genetic interaction between $p d m 3$ and acj6, and ORNs can be divided into three categories, those that depend on both of these POU genes, those that depend on acj6 alone, and those that depend on neither for the acquisition of odor specificity. These results are consistent with a combinatorial code of POU genes. $p d m 3$ is expressed in ORN classes in which it is not required for odor response, and in at least two of these classes $p d m 3$ is required for normal axon targeting. $p d m 3$ thus functions in both of the processes that dictate the precise organization of the Drosophila olfactory system: the expression of individual odor receptors and the targeting of individual glomeruli.

\section{Materials and Methods}

Drosophila stocks. The $p d m 3$ mutant was obtained from the Bloomington Stock Center (PBac $\{W H\} p d m 3^{f 00828}$ ) (Thibault et al., 2004) and contains a $10 \mathrm{~kb}$ insertion of the piggyBac $\{\mathrm{WH}\}$ transposon (for location, see Fig. $1 B$ ). The insertion location was verified using inverse PCR and 
DNA sequencing of the surrounding genomic DNA. Homozygous adults show reduced mobility and a darkened cuticle, and have a shortened life span, with an average adult survival time of $4 \mathrm{~d}$. To maximize yield of pdm3 mutant adults, culture vials were passed every 3-4 d, and a Kimwipe was inserted into vials when larvae grew to the third instar stage. $p d m 3$ mutants were then collected immediately after eclosion and placed in fresh vials for $1-2 \mathrm{~d}$ at room temperature.

As a control for the genetic background, we generated flies carrying a precise excision of the piggyBac insertion. $p d m 3$ mutant flies were crossed with flies carrying piggyBac transposase, white-eyed flies were recovered, and stocks were established. We confirmed that the excision was precise by cloning and sequencing the $p d m 3$ gene region from genomic DNA. Control flies were treated in the same manner as mutant flies The $D f$ stock was also obtained from the Bloomington Stock Center (Df(2R)Exel6058) (Parks et al., 2004). The acj6 mutant used in this study is the $a c j 6{ }^{6}$ allele, shown to be a null allele by Clyne et al. (1999b).

Reverse transcription-PCR. In an initial reverse transcription (RT)PCR, we amplified the C-terminal half of the $p d m 3$ transcript, which includes the POU domain. The following primers were used with cDNA from Canton-S heads as template: 5'-AATGAACCCACCATCAACCAG-3', 5'-GCTCTAGACTATACCATTCCCTTGGACATC-3' (Yale Keck Oligo Synthesis). The PCR products were cloned using TOPO TA Cloning (Invitrogen) and sequenced.

Electrophysiology. Extracellular single-unit recordings were performed as described by de Bruyne et al. (2001). Odorants were diluted 1:100 in paraffin oil unless otherwise indicated, and $50 \mu \mathrm{l}$ were pipetted onto a filter disc within the neck of a Pasteur pipette. A P-1000 blue micropipette tip was placed over the larger end of the pipette. The small tip of the Pasteur pipette cartridge containing the odorant was placed into a hole in a glass tube through which a purified air stream $(32 \mathrm{ml} / \mathrm{s})$ passed continuously in the direction of the fly, and the odor stimulus was delivered via a $0.5 \mathrm{~s}$ pulse of charcoal-filtered air $(3.2 \mathrm{ml} / \mathrm{s})$ through the pipette cartridge. All odor cartridges were used a maximum of three times. Responses were quantified by counting the number of spikes within the $0.5 \mathrm{~s}$ odor stimulus interval, and subtracting the number of spikes in a $0.5 \mathrm{~s}$ interval before the stimulus.

Pdm3 antibody production. A recombinant GST-Pdm3 antigen was produced, which included Pdm3 amino acids 411-766 fused to the C terminus of the GST polypeptide. The following primers were used to amplify this region from $p d m 3$ cDNA (catalog \#EDM1133-6872894, clone \#AT16994; Open Biosystems) as template: 5'-GGGAGAGGGAAAGGGAGAGAGAAC-3', 5'-GCTCTAGACTACTGCATGTGGGAAACACTCGATG-3' (Invitrogen; XbaI site indicated by boldface type). This region was chosen because it does not contain common protein motifs such as the POU domain. The product was cloned into pGEX6P-1 (GE Healthcare) and transformed into BL21 cells, which were grown at $37^{\circ} \mathrm{C}$ until $\mathrm{A}_{600}$ reached 0.8 . The fusion protein was purified from the sonicate of this culture using glutathione Sepharose $4 \mathrm{~B}$ beads (GE Healthcare) according to the manufacturer's instructions. It was stored at $4^{\circ} \mathrm{C}$ and sent to Cocalico Biologicals, where it was used to inoculate rats. The polyclonal antibody was used at a 1:100 dilution.

RNA in situ hybridization and immunohistochemistry. RNA in situ hybridizations were performed as described previously (Goldman et al., 2005). To synthesize the $p d m 3$ RNA probe, a $p d m 3$ cDNA clone in the vector pOTB7 (Open Biosystems) was used as template to amplify the gene, and all subsequent steps were performed according to the manufacturer's instructions (Roche).

Adult labella were dissected and immunostained as follows, modified from Laissue (1999) and Thorne (2004). Thirty to forty heads were dissected, placed into $500 \mu \mathrm{l}$ of fix solution (4\% paraformaldehyde in $1 \times$ PBS $+0.2 \%$ Triton X-100), and fixed for $3 \mathrm{~h}$ on wet ice. The heads were rinsed twice in wash solution $(1 \times$ PBS $+0.2 \%$ Triton $X-100)$, then washed for $1 \mathrm{~h}$ with four changes of wash solution on a rotator. The fixed heads were either stored at $4^{\circ} \mathrm{C}$ for up to 2 weeks or used immediately in immunostaining experiments as follows. Wash solution was replaced with blocking solution ( $1 \%$ BSA or $3 \%$ NGS in $1 \times$ PBS $+0.2 \%$ Triton $\mathrm{X}-100$ ), and labella were dissected in blocking solution, then blocked for $1 \mathrm{~h}$ at room temperature on a rotator. Primary antibody diluted in blocking solution [anti-Pdm3 at 1:100, anti-green fluorescent protein (GFP)
(Invitrogen rabbit anti-GFP, A6455) at 1:50, and anti-Elav (Hybridoma Bank mouse anti-Elav) at 1:10] was applied to the sample (500 $\mu \mathrm{l}$ per sample) and incubated overnight at $4^{\circ} \mathrm{C}$ on a rotator. The primary antibody mix was then saved for two more stainings, and the sample was washed three times for $\geq 30 \mathrm{~min}$ at room temperature. Secondary antibody diluted in blocking solution (1:250) was used to stain samples for $4 \mathrm{~h}$ at room temperature, in the dark and on a rotator. The secondary mix was discarded, and the sample was again washed in three 30 min washes at room temperature. Most of the wash solution was removed, and one to two drops of Vectashield Mounting Medium (Vectashield H-1000) were applied. Mounted slides were either stored at $4^{\circ} \mathrm{C}$ for a maximum of $2 \mathrm{~d}$ or immediately viewed and photographed using a Bio-Rad MRC 1024 confocal microscope. Adult brains were dissected and immunostained as previously described by Wu and Luo (2006). Slides were viewed on a Bio-Rad MRC 1024 confocal microscope.

In RNA hybridization/immunostaining double stainings, first the RNA in situ hybridization experiments were performed as described by Goldman et al. (2005), followed by antibody staining as described above.

Transgenes. To generate UAS-pdm3 cDNA constructs, the commercially available $p d m 3 \mathrm{cDNA}$ (Open Biosystems) was used as template to amplify the long $p d m 3 \mathrm{cDNA}$ splice form, with the following primers, which include restriction enzyme sites: 5'-GTACAGATCTATGGAGATCACGGACGGC-3' (BglII site indicated by boldface type), 5'-ATGCGCGGCCGCCTATACCATTCCCTTGGACATC-3' (NotI site indicated by boldface type). The PCR product was cloned into pGEM-T Easy (Promega). The short form was made by using two Bsm I (New England Biolabs) sites that flank the alternatively spliced region to swap the long splice form DNA out of the construct and replace it with short splice form DNA, which had been cloned previously into the pCRII TOPO vector (Invitrogen) in initial RT-PCR analysis. Clones were sequenced, and those clones with the correct sequence were swapped into pUAST (Invitrogen) using the restriction enzymes BglII and NotI (New England Biolabs).

For all experiments using GFP, a UAS-mCD8-GFP construct was used (Lee and Luo, 1999), and the GFP was detected with the anti-GFP antibody.

\section{Results}

\section{Identification of a new Drosophila POU gene, pdm3}

We identified a new POU gene by searching the Drosophila genome sequence with POU domain sequences from all six POU gene classes [for review of POU classes, see Ryan and Rosenfeld (1997)]. The new gene belongs to class VI, and we named it $p d m 3$ (POU domain motif 3 ) by analogy to the previously named $p d m 1$ and $p d m 2$ genes. This is the first POU class VI gene in Drosophila, and we found no other members of this class in the fly genome.

The POU domain of $p d m 3$ consists of a POU-specific domain, a POU-homeodomain, and a linker region that separates the two (Fig. 1A). It is located in the C-terminal part of the gene, as in other POU genes of class VI. $p d m 3$ spans $\sim 8 \mathrm{~kb}$ and contains 10 exons (Fig. $1 \mathrm{~B}$ ). The predicted POU domain is the most highly conserved region of the gene, showing $70-90 \%$ identity with other class VI POU proteins and $40-50 \%$ identity with POU proteins of other classes (supplemental Fig. S1, available at www.jneurosci.org as supplemental material).

In initial RT-PCR experiments, we amplified $p d m 3$ from adult head RNA. Two splice forms were amplified, henceforth called the "long" and "short" forms. They differ in that the long form includes exon 7, which is missing from the short form (Fig. $1 B, C)$. This exon encodes 18 aa that interrupt the POU domain. An optional exon has likewise been found at the same location in Rpf-1, a human ortholog of $p d m 3$ (Zhou et al., 1996), and other class VI POU genes (supplemental Fig. S1, available at www. jneurosci.org as supplemental material). 


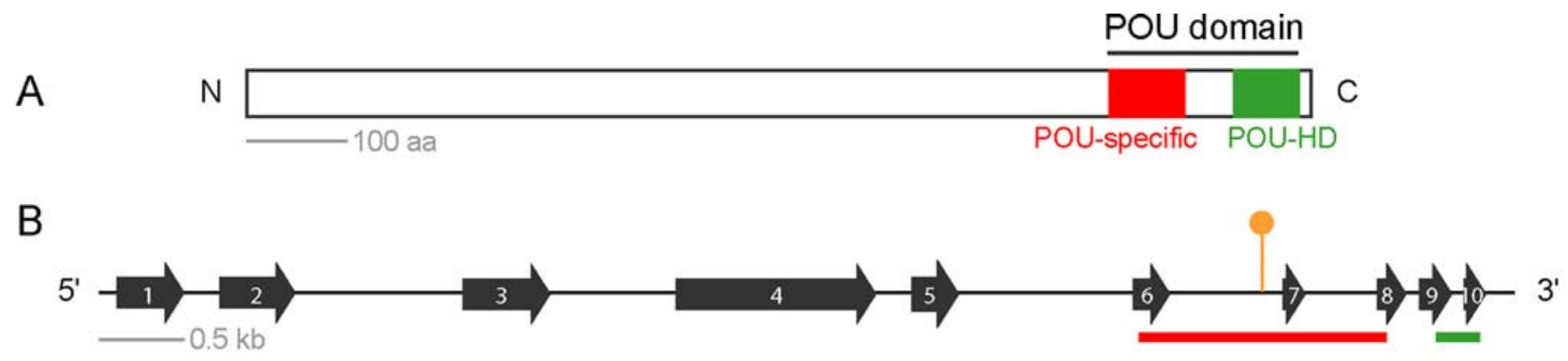

C

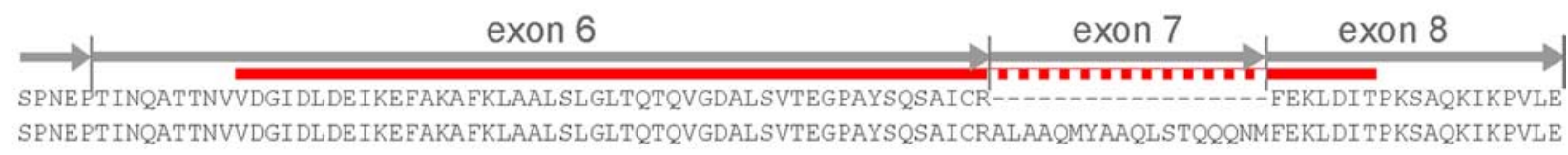

short SPNEPTINQATTNVVDGIDLDEIKEFAKAFKLAALSLGLTQTQVGDALSVTEGPAYSQSAICR-_-_._._. long SPNEPTINQATTNVVDGIDLDEIKEFAKAFKLAALSLGLTQTQVGDALSVTEGPAYSQSAICRALAAQMYAAQLSTQQQNMFEKLDITPKSAQKIKPVLE

exon 9

exon 10

short RWMKEAEESHWMRYKSGNHLTDVIGBEPSKKRKAATSETPQRLELLNA'HFEANTHPSGTEITGLAHQLGVEREVIRIQECNKRQALKNTVAMMSKGMVN long RWMKEAEESHWMRYKSQNHLTDVIGBEPSKKRKAATSFT PQRLELLNAHFEANTHPSGTEITGLAHQLGVEREVIRIQFCNKRQALKNTVAMMSKGMVN

Figure 1. The structure of $p d m 3$. $A$, The POU domain, with the POU-specific domain (red) and POU homeodomain (green) indicated. $\boldsymbol{B}$, The genomic organization of $p d m 3$. The transposon insertion (orange symbol) is located in an intron between two exons that encode the POU-specific domain (red bar). C, Optional splicing of exon 7. The region of the POU domain indicated by a dashed red bar is excluded from the short splice form but included in the long splice form.

\section{$p d m 3$ is expressed in ORNs}

The fruit fly detects odors with two types of olfactory organ, the antenna and the maxillary palp (Fig. $2 A$ ). In situ hybridization experiments showed that $p d m 3$ is expressed in a subset of cells in the antenna (Fig. $2 B$ ). Expression was also detected in the maxillary palp, where we performed a double-label experiment to determine whether expression was neuronal. We found that cells that express $p d m 3$ RNA are labeled by an antibody against Elav, which labels the nuclei of differentiated neurons (Fig. 2C).

To characterize the expression of $p d m 3$ in more detail, we then generated an antibody, using as antigen a portion of the protein that did not contain POU domain sequences. The antibody labeled cells in the maxillary palp (Fig. 2D). All or almost all of these Pdm $3^{+}$cells are Elav ${ }^{+}$(Fig. 2E). Many, but not all, of the $\mathrm{Elav}^{+}$cells are $\mathrm{Pdm} 3^{+}$. In the Pdm ${ }^{+} \mathrm{Elav}^{+}$cells, the labeling appeared coincident. Together, our results show that $p d m 3$ is expressed in the nuclei of a subset of maxillary palp neurons.

We obtained a mutant line that contains a transposon insertion in $p d m 3$ (Thibault et al., 2004). The insertion lies within an intron between two exons that encode the POU domain (Fig. $1 B$ ). This insertion eliminated $\mathrm{Pdm} 3$ expression, indicating specificity of the antibody (Fig. 2F). The insertion did not markedly reduce the number of Elav ${ }^{+}$cells (Fig. $2 G$ ), suggesting that the loss of $p d m 3$ does not cause a loss of neurons.

\section{A defect in odor response in the pdm 3 maxillary palp}

To determine whether $p d m 3$ is required for the function of ORNs, we measured ORN responses to odors using single-unit electrophysiology. The antenna and maxillary palp contain a number of functional types of sensilla, as defined by electrophysiological and molecular analysis (de Bruyne et al., 1999, 2001; Couto et al., 2005; Goldman et al., 2005). Each sensillum type contains up to four ORNs, usually two, which are combined in a stereotyped configuration. The maxillary palp is the simpler organ, in that it contains only three functional types of sensilla, pb1, $\mathrm{pb} 2$, and $\mathrm{pb} 3$, each of which contains a pair of ORNs: pb1A and pb1B; pb2A and pb2B; pb3A and pb3B. Odorants pass through
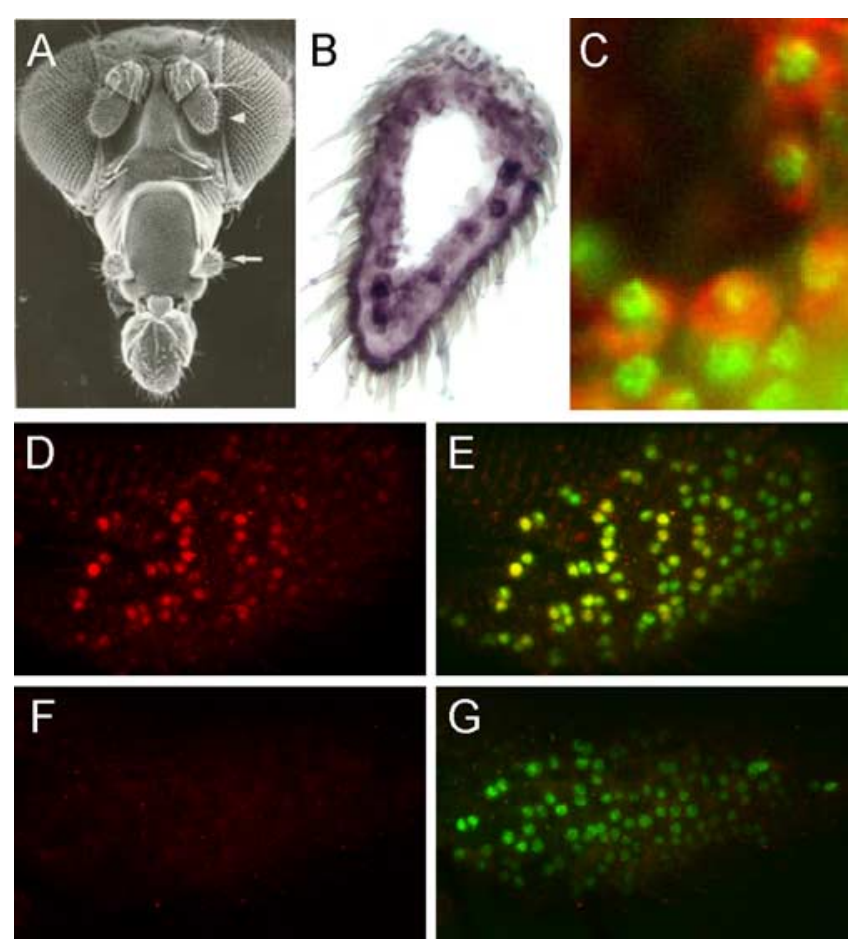

Figure 2. $\quad p d m 3$ is expressed in neurons of the olfactory system. A, Drosophila olfactory organs. The third segment of the antenna is indicated by an arrowhead, and the maxillary palp is indicated by an arrow [reprinted with permission from Carlson (1996), his Fig. 1]. $\boldsymbol{B}, p d m 3$ in situ hybridization to the antenna shows expression in a subset of cells, in blue. $C, p d m 3$-expressing cells of the maxillary palp, stained by an RNA probe in red, are also stained in the nucleus by anti-Elav (green), a marker for mature Drosophila neurons. $\boldsymbol{D}, \boldsymbol{E}$, A control maxillary palp stained with anti-Pdm3 in $\operatorname{red}(\boldsymbol{D}, \boldsymbol{E})$ and anti-Elav in green $(\boldsymbol{E}) . \boldsymbol{F}$, $\boldsymbol{G}$, A pdm3 maxillary palp stained with anti-Pdm3 in $\operatorname{red}(\boldsymbol{F}, \boldsymbol{G})$ and anti-Elav in green $(\boldsymbol{G})$. The nuclear anti-Pdm3 staining visible in neurons of the control maxillary palp is lost in the mutant. The control used here and in the remainder of this study is a precise excision of the transposon insertion. 
A control

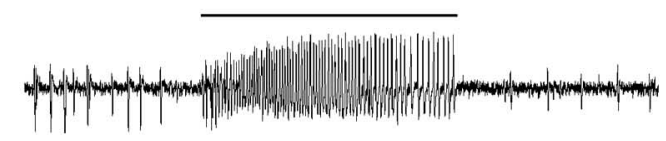

pdm3

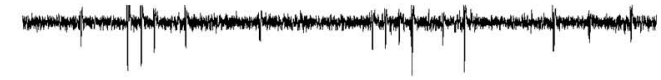

B
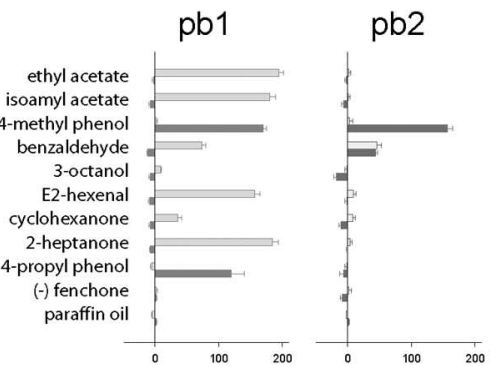

$\mathrm{pb} 3$
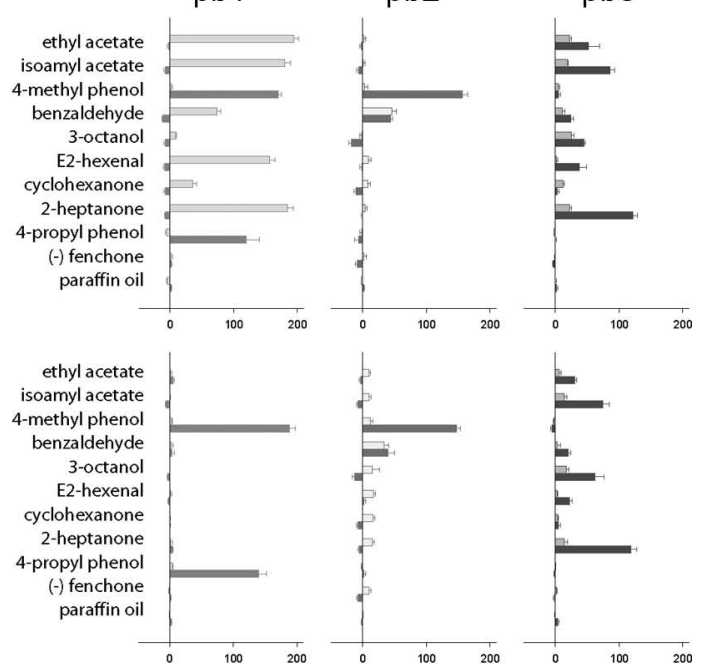

$p d m 3$
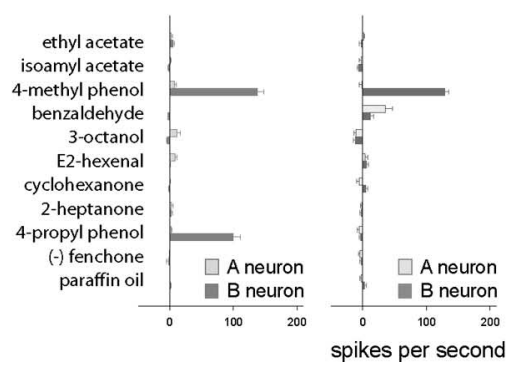

spikes per second

Figure 3. An olfactory defect in the $p d m 3$ maxillary palp. $\boldsymbol{A}$, Electrophysiological responses from control and mutant pb1 sensilla. Spikes, or action potentials, of large amplitude are produced by the A neuron; spikes of small amplitude are produced by the $B$ neuron. The control pb1A neuron responds to a $0.5 \mathrm{~s}$ stimulus of E2-hexenal, indicated by the bar. Although both neurons are present in the mutant $\mathrm{pb} 1$ sensillum, neither responds to the odor stimulus. $\boldsymbol{B}$, Responses of neurons in spikes per second ( $n \geq 11$; error bars represent SEM). pb1 sensilla were identified by the response of the neighboring $\mathrm{ORN}$ in the sensillum to 4-propyl phenol.

pores in the sensillum walls, traverse the internal lymph, and activate the ORNs. Seven Or genes are expressed in the maxillary palp: of the six ORN classes, each expresses one odor receptor, except that one class, pb2A, expresses two.

We found that of the six ORN classes of the maxillary palp, one is severely defective in $p d m 3$. The pb1A ORN, which in wild type responds to a pulse of E2-hexenal with a train of action potentials, does not respond in $p d m 3$ (Fig. $3 A$ ). pb1A is present and yields spontaneous action potentials, but does not respond to the odorant.

Systematic measurement of neuronal responses, measured in action potentials/s, after stimulation with a diverse panel of odorants, revealed that $\mathrm{pb} 1 \mathrm{~A}$ failed to respond to any tested odorant. In contrast, in the same sensillum, the neighboring pb1B ORN responded normally to 4-methyl phenol and 4-propyl phenol, its most effective odorants. Neurons of the $\mathrm{pb} 2$ and $\mathrm{pb} 3$ sensilla gave very similar responses in $p d m 3$ and control animals (Fig. $3 B$ ).

As a more stringent test of the role of $p d m 3$ in ORN response, we tested the $p d m 3$ mutation in heterozygous combination with a deletion for the region. We found that in this $p d m 3 / D f$ heterozygote, all responses were very similar to those of the $p d m 3$ homozygote, indicating that the $p d m 3$ insertion is a null allele (Fig. $3 B)$.

The olfactory phenotype is not limited to the maxillary palp. We examined the large basiconic sensilla of the antenna and found that one of the four ORNs in the ab1 sensillum, ab1A, is present but has lost odor response (supplemental Fig. S2, available at www.jneurosci.org as supplemental material). Limited analysis of the ORNs in ab2 and ab3 sensilla revealed no abnormalities. The detection of an olfactory phenotype in some antennal ORNs is consistent with our detection of $p d m 3$ expression in a subset of antennal cells (Fig. 2B).

\section{A defect in odor receptor gene expression}

Because pb1A is present in $p d m 3$ and yields spontaneous action potentials, but does not respond to odors, we wished to test the hypothesis that $p d m 3$ affects expression of its odor receptor. Or42a is the odor receptor that is expressed in pb1A (Goldman et al., 2005); a receptor-to-neuron map of the maxillary palp is shown in Figure $4 A$.

As a first test of this hypothesis, we asked whether Pdm3 is expressed in pb1A in wild type. A double-label experiment showed that Pdm3 is in fact expressed in Or42a-expressing cells of wild type (Fig. $4 B$ ). We then found that Or42a expression is undetectable in $p d m 3$ (Fig. $4 C$ ). In contrast, the neighboring $\mathrm{pb} 1 \mathrm{~B}$ cell shows normal expression of its receptor gene, Or71a, and all other maxillary palp odor receptor genes are expressed in pdm3 (Fig. 4C). Thus these expression studies coincide precisely with the functional studies: the one class of ORN that has lost function is the one class of ORN that has lost expression of a receptor gene.

\section{Rescue of olfactory response by $\mathrm{Or} 42 a$ and $p d m 3$}

To determine whether the loss of pb1A odor response is attributable solely to loss of Or $42 a$ expression or whether other essential components are lost as well, we drove expression of Or42a in pb1A neurons of $p d m 3$ using the GAL4-UAS system. We tested response to the two odorants that elicit the strongest response from pb1A and found that responses to both were completely or largely restored when Or42a was driven by Elav-GAL4 (Fig. $5 A, B)$. These results suggest a surprising degree of specificity to the $p d m 3$ phenotype at the molecular level.

We next investigated the ability of $p d m 3$ splice forms to rescue the phenotype. Of the two splice forms, we amplified only the long form from adult maxillary palps in RT-PCR experiments (data not shown). When we drove expression of a long form cDNA using the GAL4-UAS system, we were able to rescue the phenotype of pb1A completely or largely in 7 of $29 \mathrm{pb} 1$ sensilla, tested with three odorants (Fig. $5 \mathrm{C}$ ). Expression of Or $42 a$ was also restored in a number of cells (Fig. $5 D$ ). In this experiment, we used an acj6-GAL4 driver (Bourbon et al., 2002), which drives expression in all or almost all maxillary palp ORNs. We were unable to construct a faithful driver for $p d m 3$, whose $5^{\prime}$ end is separated from the next annotated upstream gene by a long region; it is possible that a $p d m 3$ driver might restore the pb1A phenotype in a greater fraction of pb1 sensilla. We also drove expression of a short form cDNA and found rescue in 1 of $19 \mathrm{pb} 1$ sensilla tested (data not shown).

We note that in addition to the rescued pb1A cells, we also found a number of pb1A cells that acquired an odor response profile different from that of wild type. Further testing revealed 
A

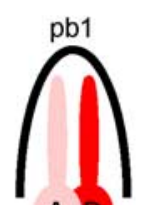

A

Or42a Or71a

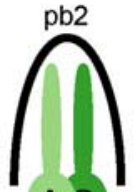

A B

Or 85 e Or 46 a

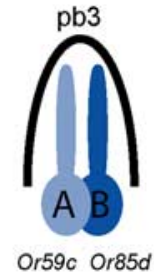

Or59c Or85c
B

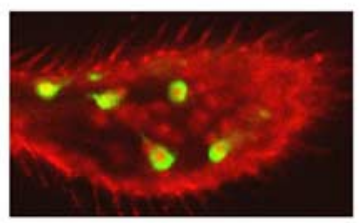

C

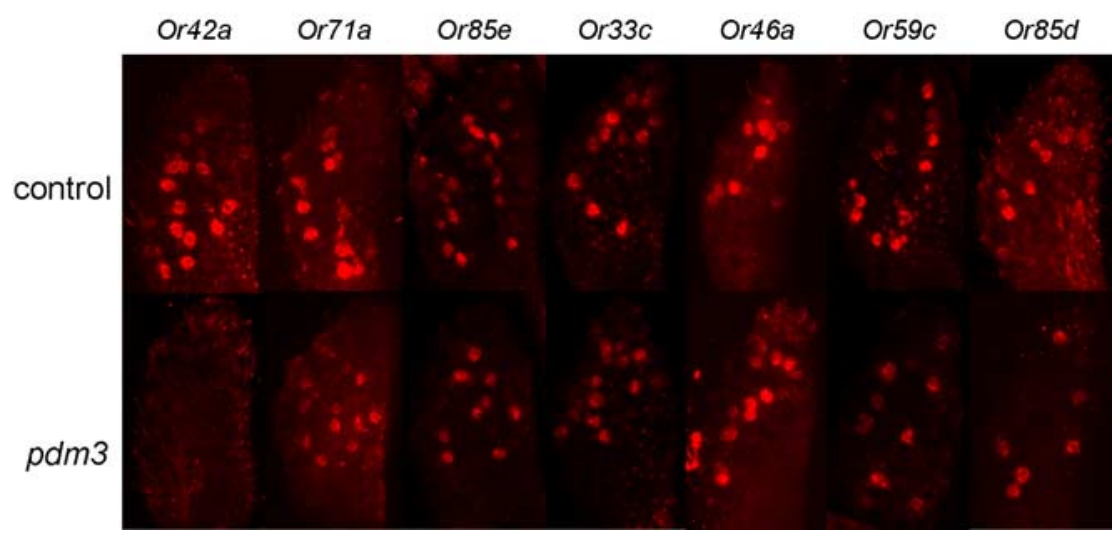

Figure 4. Gene expression in the $p d m 3$ maxillary palp. $\boldsymbol{A}, \mathrm{A}$ receptor-to-neuron map of the maxillary palp. $\boldsymbol{B}, p d m 3$ is expressed in the pb1A neuron class. pb1A neurons were labeled in green using Or42a-GAL4; UAS-mCD8GFP, and pdm3expressing cells were labeled in red with the Pdm3 antibody. All labeled pb1A neurons are labeled with the Pdm3 antibody. $C$, RNA in situ hybridizations for each of the maxillary palp Or genes in control and mutant.

that this odor response profile is very similar to that conferred by a larval odor receptor, Or85c (supplemental Fig. S3A, available at www.jneurosci.org as supplemental material) (Kreher et al., 2005). Moreover, Or85c expression is detected in several cells of the "rescued" maxillary palp (supplemental Fig. S3B , available at www.jneurosci.org as supplemental material). The proportion of cells with this response profile is greater when $p d m 3$ is driven with a promoter that is expected to drive later expression in ORNs (supplemental Fig. S3C, available at www.jneurosci.org as supplemental material). Further testing beyond the scope of this study would be required to determine whether these results reflect the presence of different $p d m 3$ interaction partners at different times at the promoters of Or $42 a$ and Or85c.

\section{A genetic interaction between $p d m 3$ and acj6 in the pb1A neuron}

Because pb1A is affected by both $p d m 3$ and another POU gene, acj6 (Clyne et al., 1999b), we investigated the relationship between them. Although the concept of a combinatorial code of transcription factors has been previously invoked in discussion of receptor gene choice in Drosophila (Ray et al., 2007, 2008), the interaction of two transcription factors within the same ORN has not yet been examined in Drosophila.

We found first that neither POU gene appears to regulate the other in the maxillary palp: $p d m 3$ is expressed in a null mutant of acj6, and acj6 is expressed in a null mutant of $p d m 3$ (Fig. $6 B, C$ ). We then found that although both $p d m 3$ and acj6 are fully recessive, the transheterozygote shows a reduced response of pb1A (Fig. 6D). The response of pb1B is normal in this genotype. These results indicate a genetic interaction between the two POU genes in pb1A.

Interestingly, two putative $\mathrm{Pdm} 3$ binding sites, i.e., sethe possibility that $p d m 3$ might have a different function in these cells.

\section{$p d m 3$ is required for axon targeting of two ORN classes}

ORNs send axons to the antennal lobe of the brain, and axons of an individual ORN class converge precisely on an individual unit of the antennal lobe called a glomerulus (Fig. 8A) (Gao et al., 2000; Vosshall et al., 2000; Couto et al., 2005; Fishilevich and Vosshall, 2005). Thus, a spatial map of olfactory information is created in the antennal lobe by the stereotyped pattern of connectivity. There are 43 glomeruli in the antennal lobe, and both receptor-to-ORN and ORN-to-glomerulus maps have been constructed (Hallem et al., 2004; Couto et al., 2005; Fishilevich and Vosshall, 2005; Goldman et al., 2005). A number of genes required for normal ORN axon targeting have been identified (Komiyama and Luo, 2006).

We examined the axon targeting of two ORN classes that express $p d m 3$ but that do not require $p d m 3$ for odor response: $\mathrm{pb} 1 \mathrm{~B}$, which expresses the Or71a receptor, and pb3A, which expresses $\operatorname{Or} 59 \mathrm{c}$. We found that in a $p d m 3$ mutant, the axonal projections of both ORN classes are abnormal (Fig. $8 B$ ). In the wild type, in both cases the projections converge on a single, discrete glomerulus in each antennal lobe. In $p d m 3$, the convergence is less precise and the boundaries of the targeted areas are less discrete. In some cases, the axon tracts appear to bifurcate, with labeled axons occupying more than one region. In most cases, the axons appear to terminate in the general vicinity of the wild-type target, but not in the precise positions of the wild-type glomerular targets. In summary, $p d m 3$ has effects on ORN axon targeting, in addition to its role in specifying odor response and receptor gene expression. 

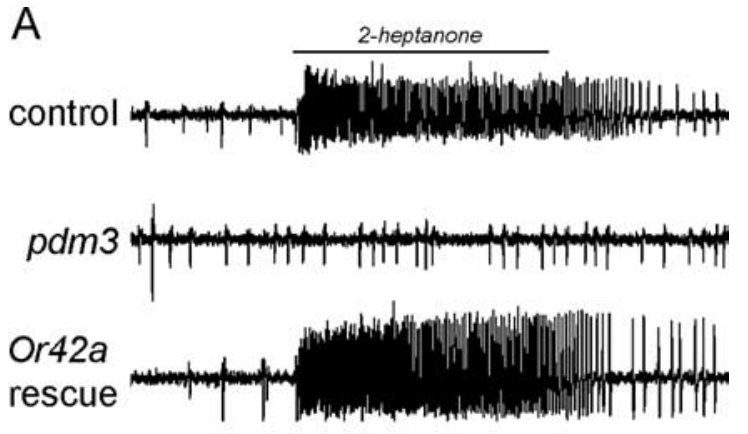

B

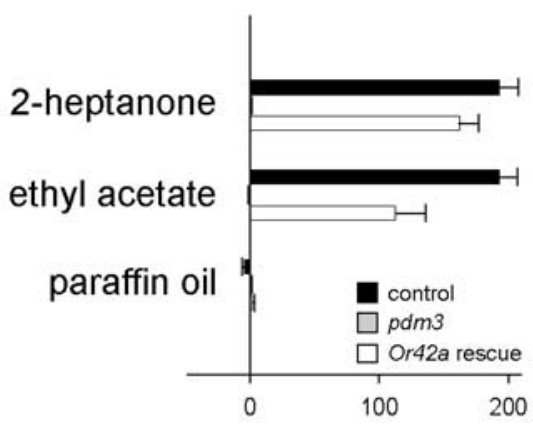

spikes per second

C

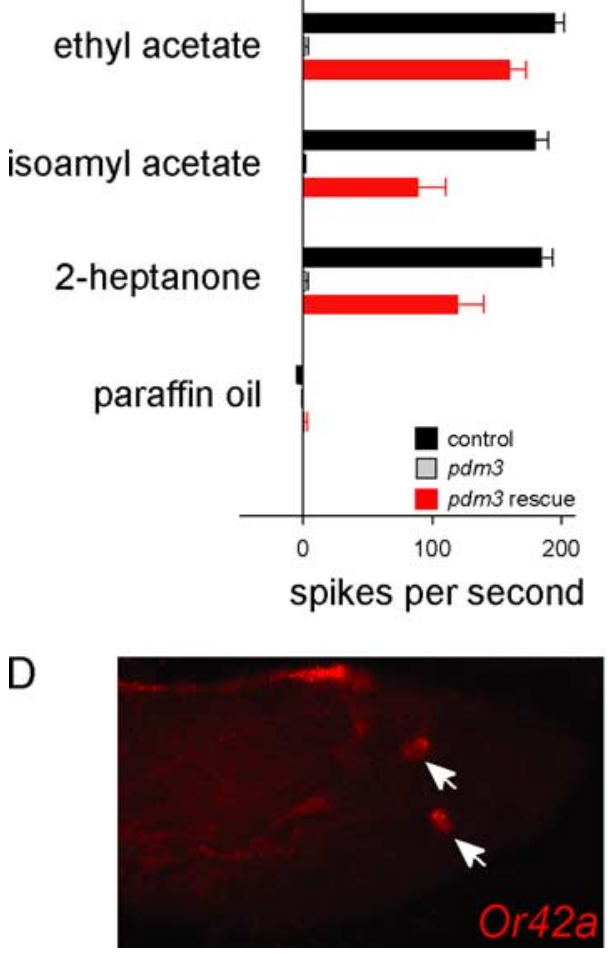

Figure 5. Rescue of pb1A odor response. $A$, The response to a $0.5 \mathrm{~s}$ pulse of 2 -heptanone is restored by expression of Or42a in the $p d m 3$ mutant. Or42a was expressed in the $p d m 3$ background using (155(elav)-GAL4; UAS-Or42a. B Rescue of pb1A responses (white) to the two odors that activate pb1A the most strongly. The large-amplitude spikes of the A neuron were counted. $n=6$; error bars represent SEM. C, Rescue with a $p d m 3$ CDNA, using acj6-GAL4 and UAS-pdm 3 CDNA (long isoform) in the mutant background. $n=6 . D$, In situ hybridization shows expression (arrows) of Or42a in maxillary palps of acj6-GAL4/w; pdm3, UAS-pdm3.
A
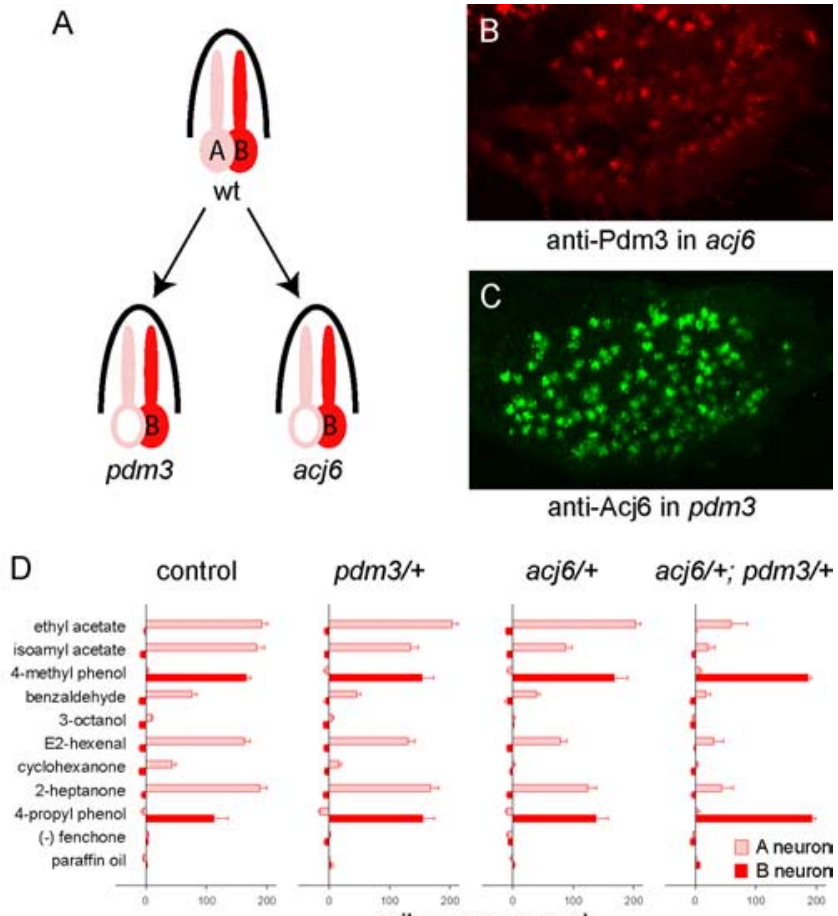

anti-Pdm3 in acj6

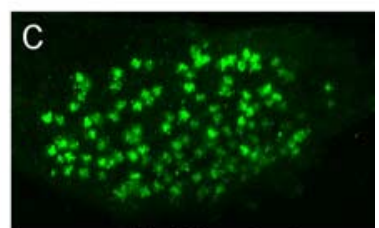

anti-Acj6 in pdm3

spikes per second

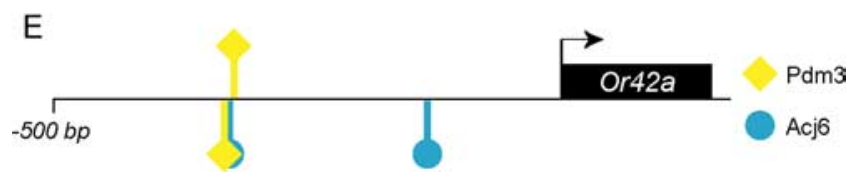

Figure 6. A genetic interaction between $p d m 3$ and acj6 in pb1A. $\boldsymbol{A}$, The pb1A neuron is affected by both $p d m 3$ and acj6. $\boldsymbol{B}$, Anti-Pdm3 immunostaining of acj6 $\sigma^{6}$ maxillary palps. $\boldsymbol{C}$, Anti-Acj6 immunostaining of $p d m 3$ maxillary palps. $\boldsymbol{D}$, pb1A responses are reduced in the transheterozygote. pb1A responses of each single heterozygote appear comparable with control responses. $n \geq 7$; error bars $=$ SEM. $\boldsymbol{E}$, Putative Pdm3 and Acj6 binding sites upstream of Or42a. One putative Pdm 3 binding site overlaps with an Acj6 site, and another lies immediately adjacent.

\section{Discussion}

The development of the olfactory system depends on two intriguing processes. ORNs must select which odor receptors to express and which glomeruli to target in the brain. Little is known about the molecular mechanisms underlying either process.

We have identified a new Drosophila POU gene, $p d m 3$, that acts in both receptor gene expression and axonal targeting. Pdm3 is a POU transcription factor of class VI. Vertebrate members of class VI are expressed in brain and spinal cord (Andersen et al., 1993; Johansen et al., 1993; Okamoto et al., 1993; Wey et al., 1994; Zhou et al., 1996). Notably, a mouse ortholog of $p d m 3$, Emb, is expressed in the olfactory bulb (Wey et al., 1994).

The mechanism of ORN fate determination has been elegantly examined in Caenorhabditis elegans. The AWA, AWB, and AWC cells express multiple olfactory receptors and sense distinct but overlapping sets of odors (Bargmann et al., 1993). The specification of AWA fate requires lin-11, a LIM homeodomain gene (Sarafi-Reinach et al., 2001). lin-11 regulates ODR-7, a nuclear hormone receptor that promotes expression of AWA-specific genes and represses AWC-specific genes (Sengupta et al., 1994). The Aristaless-related homeodomain gene alr-1 is also required for specification of AWA (Melkman and Sengupta, 2005). ceh-37, an Otx homeodomain gene, regulates lim-4, another LIM homeobox gene, to promote AWB neuron fate (Sagasti et al., 1999; 
A pb1A pb1B pb2A pb2B pb3A
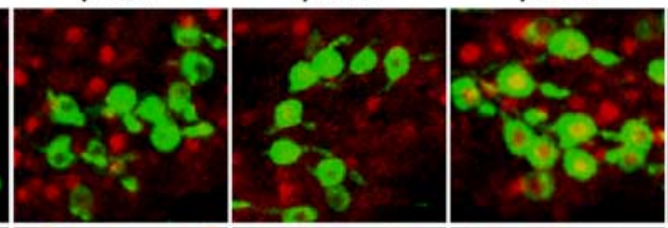

pb3B
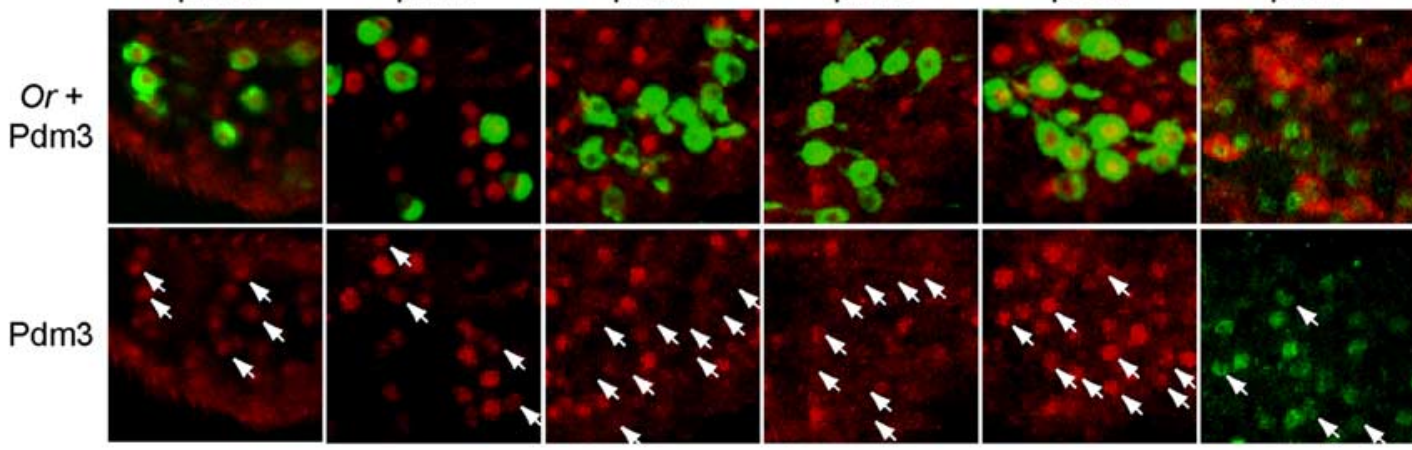

Pdm3
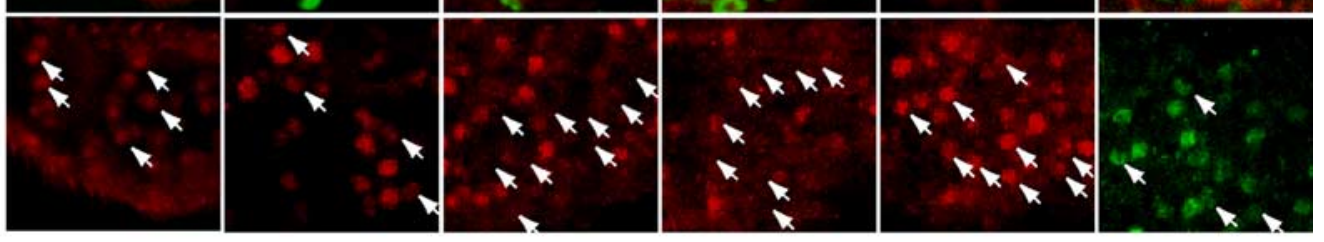

B

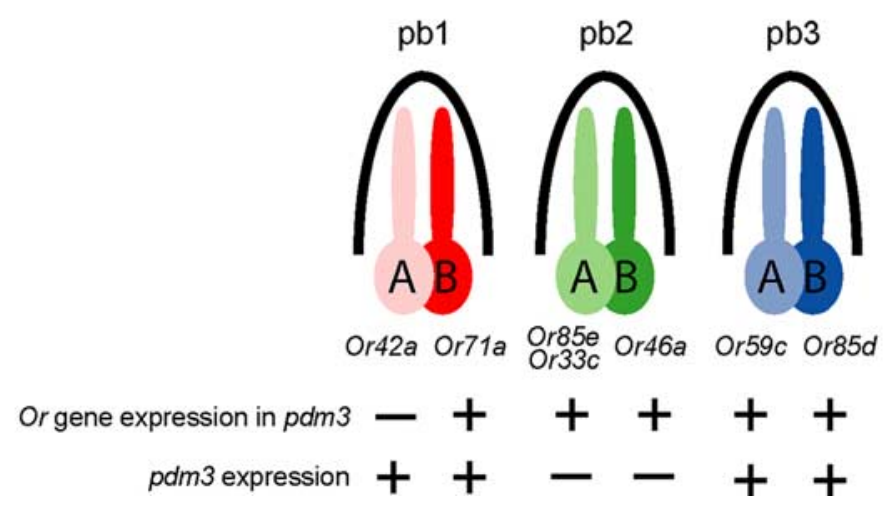

Figure 7. $p d m 3$ is expressed in four maxillary palp ORN classes. $A$, The pb1A, pb1B, pb2A, pb2B, and pb3A neuron classes were labeled in green using the GAL 4 system to drive GFP, and in these cases pdm3-expressing cells were labeled in red using the Pdm3 antibody. The pb3B neuron class was labeled in red using an Or85d in situ hybridization probe, and in this case $p d m 3$-expressing cells were labeled in green with the Pdm3 antibody. Arrows in the bottom panels indicate the location of ORNs that are labeled in the top panels. Anti-Pdm3 staining overlaps with that of pb1A, pb1B, pb3A, and pb3B, but not with that of pb2A and pb2B. $B$, Summary. One Or gene is affected in $p d m 3$, and $p d m 3$ is expressed in four maxillary palp 0RN classes.

A

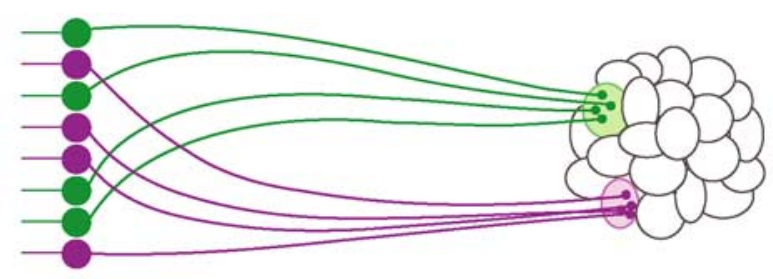

B
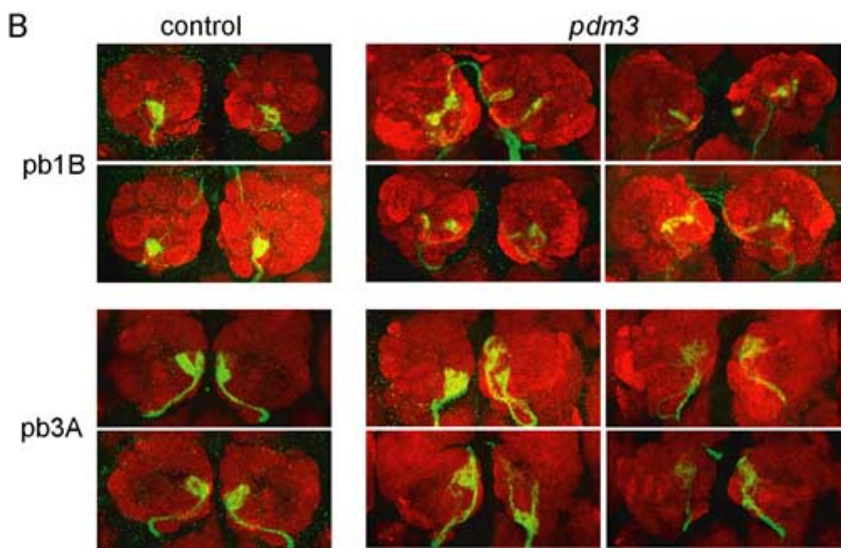

Figure 8. $p d m 3$ has effects on ORN axon targeting. $\boldsymbol{A}, \mathrm{ORN}$ axons of a given class, represented in a common color (green or purple), project to the same glomerulus of the antennal lobe. $\boldsymbol{B}$, Targeting of the pb1B (green, Or71a-GAL4; UAS-GFP) and pb3A (green, Or59c-GAL4; UAS-GFP) ORN classes are affected by pdm3. Neuropil is stained in red with anti-nc82.
Lanjuin et al., 2003). Another Otx gene, ceh-36, is required for AWC neuron fate (Lanjuin et al., 2003).

The first olfactory phenotype we detected for $p d m 3$ was its defective odor response in an electrophysiological assay. The phenotype is striking in its specificity, at both the cellular and molecular levels. At the cellular level, only one ORN class in the maxillary palp, pb1A, was defective in a null mutant of $p d m 3$. The pb1B neuron, which neighbors pb1A in the same sensillum, and the other four ORN classes all appeared to yield normal odor responses. At the molecular level, the pb1A physiological phenotype appeared to be attributable largely if not completely to the loss of a single gene: Or42a, the odor receptor gene normally expressed in this neuron. Moreover, Or $42 a$ is the only maxillary palp Or gene affected by $p d m 3$.

Although we were surprised by the specificity of the molecular and cellular phenotypes, the specificity of one phenotype is in good agreement with the specificity of the other. The cascade of molecular steps between the presentation of an odor molecule and the production of an action potential is poorly understood but presumably requires the agency of a number of different genes. It seems likely, however, that the signaling pathway used by one maxillary palp ORN class is the same as that used by the others, with the exception of the odor receptor. Thus the ability to rescue the pb1A odor response with its receptor gene alone supports a model in which the rest of the signaling pathway does not depend on $p d m 3$. In this case, $p d m 3$ would affect odor response only in those maxillary palp ORNs whose receptor genes it affects, i.e., only in pb1A.

The specificity of $p d m 3$ is in contrast to that of acj6, which affects the odor response of four ORN classes in the maxillary 
palp (Clyne et al., 1999b). Moreover, in acj6 some of the unresponsive ORNs were not detected electrophysiologically, either because of a defect that made them physiologically silent or because they were absent. Thus acj6 appears to affect more ORNs and to affect some of them more severely than $p d m 3$. We note the formal possibility that $p d m 3$ acts in some ORNs other than pb1A but is functionally redundant in them.

The requirement of both $p d m 3$ and acj6 in pb1A allowed us to investigate whether they act together or independently. We found a genetic interaction between these two POU genes in our analysis of pb1A odor response. The location of overlapping putative binding sites for both transcription factors upstream of Or $42 a$ suggests the possibility of a biochemical interaction between them. Heterodimerization, one possible means of interaction, has been shown previously for other POU proteins (Voss et al., 1991). The genetic interaction and coexpression of acj6 and $p d m 3$ in ORNs is in contrast to the relationship between acj6 and another POU gene, drifter, in projection neurons (PNs), the postsynaptic partners of ORNs. Although both acj6 and drifter act in PNs, they are expressed in mutually exclusive subsets of PNs.

A major problem in olfactory system biology is how individual ORNs select which of a large family of odor receptor genes to express. There are 60 Or genes in the genome of Drosophila melanogaster (Clyne et al., 1999a; Vosshall et al., 1999; Robertson et al., 2003). The expression of particular Or genes, and hence the odor specificity of the ORN, has recently been found to depend on a regulatory code of cis-acting elements. Positive and negative regulatory elements named Dyad-1 and Oligo- 1 are required for the selection of $\mathrm{Or}$ genes in the correct olfactory organ (Ray et al., 2007). Within the maxillary palp, additional elements act positively to promote expression of individual Or genes in a subset of ORN classes, whereas other elements act negatively to restrict expression of individual Or genes to a single ORN class (Ray et al., 2007,2008 ). We also found evidence that a combinatorial code of transcription factors underlies the problem of receptor gene choice (Ray et al., 2007). Transcription factors that act in this process include Lozenge, a Runx domain-containing protein that is required for the expression of two Or genes (Ray et al., 2007), and Scalloped, which mediates repression (Ray et al., 2008). Another subset of maxillary palp Or genes depends on the POU gene acj6.

Our current finding that two POU genes, acj6 and $p d m 3$, are required in the same ORN for receptor gene expression suggests that the combinatorial action of POU genes may be an important part of such a code. The Or genes of the maxillary palp can be divided into three classes: those that require both POU genes (Or42a), those that require only acj6 (Or85e, Or33c, Or46a, and Or59c), and those that require neither (Or71a and Or85d). Heterodimer formation and alternative splicing could expand the number of components that act in selecting individual receptors from the entire family of 60 Or genes in the entire olfactory system, including the ORNs of the antenna.

We found that $p d m 3$ is also required for axon targeting of pb1B and pb3A cells. Interestingly, acj6 is also required for axon targeting of these ORNs (Komiyama et al., 2004), but we do not know whether the two POU proteins act together on common transcriptional target genes or independently on different genes required for axonal wiring.

The relationship between receptor expression and ORN axon targeting has been a topic of great interest. In vertebrates, there is evidence that the odor receptor acts in both processes (Mombaerts et al., 1996; Wang et al., 1998; Feinstein and Mombaerts, 2004). In Drosophila, the receptor does not appear to be required for normal axon targeting (Dobritsa et al., 2003; Wang et al., 2003). Likewise, the effects of $p d m 3$ on receptor expression and axon targeting appear to be separable, in that pb1B and $\mathrm{pb} 3 \mathrm{~A}$ show apparently normal receptor gene expression but abnormal targeting. A related issue is the relationship between ORN activity and axon targeting. In vertebrates, there is evidence that ORN activity is necessary for the establishment or maintenance of correct ORN axon targeting (Zhao and Reed, 2001; Imai et al., 2006; Serizawa et al., 2006). We were unable to ask whether in $p d m 3$ the lack of odor response in pb1A correlates with a failure in axon targeting, for lack of a pb1A GAL4-driver that functions normally in the absence of $p d m 3$. However, we found that the normal odor responses of $\mathrm{pb} 1 \mathrm{~B}$ and $\mathrm{pb} 3 \mathrm{~A}$ ORNs are not sufficient for normal axon targeting.

This is the first mutational analysis of a class VI POU gene and demonstrates the essential role that $p d m 3$ plays in the development of a highly complex and precisely organized sensory system. Further study of $p d m 3$ may uncover critical roles in other systems as well.

\section{References}

Andersen B, Schonemann MD, Pearse RV 2nd, Jenne K, Sugarman J, Rosenfeld MG (1993) Brn-5 is a divergent POU domain factor highly expressed in layer IV of the neocortex. J Biol Chem 268:23390-23398.

Ayer RK Jr, Carlson J (1991) acj6: a gene affecting olfactory physiology and behavior in Drosophila. Proc Natl Acad Sci U S A 88:5467-5471.

Bargmann CI, Hartwieg E, Horvitz HR (1993) Odorant-selective genes and neurons mediate olfaction in C. elegans. Cell 74:515-527.

Bourbon H-M, Gonzy-Treboul G, Peronnet F, Alin M-F, Ardourel C, Benassayag C, Cribbs D, Deutsch J, Ferrer P, Haenlin M, Lepesant J-A, Noselli S, Vincent A (2002) A P-insertion screen identifying novel X-linked essential genes in Drosophila. Mech Dev 110:71-83.

Carlson JR (1996) Olfaction in Drosophila: from odor to behavior. Trends Genet 12:175-180.

Clyne PJ, Warr CG, Freeman MR, Lessing D, Kim J, Carlson JR (1999a) A novel family of divergent seven-transmembrane proteins: candidate odorant receptors in Drosophila. Neuron 22:327-338.

Clyne PJ, Certel SJ, de Bruyne M, Zaslavsky L, Johnson WA, Carlson JR (1999b) The odor specificities of a subset of olfactory receptor neurons are governed by Acj6, a POU-domain transcription factor. Neuron 22:339-347.

Couto A, Alenius M, Dickson BJ (2005) Molecular, anatomical, and functional organization of the Drosophila olfactory system. Curr Biol 15:1535-1547.

de Bruyne M, Clyne PJ, Carlson JR (1999) Odor coding in a model olfactory organ: the Drosophila maxillary palp. J Neurosci 19:4520-4532.

de Bruyne M, Foster K, Carlson JR (2001) Odor coding in the Drosophila antenna. Neuron 30:537-552.

Dobritsa AA, van der Goes van Naters W, Warr CG, Steinbrecht RA, Carlson JR (2003) Integrating the molecular and cellular basis of odor coding in the Drosophila antenna. Neuron 37:827-841.

Feinstein P, Mombaerts P (2004) A contextual model for axonal sorting into glomeruli in the mouse olfactory system. Cell 117:817-831.

Fishilevich E, Vosshall LB (2005) Genetic and functional subdivision of the Drosophila antennal lobe. Curr Biol 15:1548-1553.

Gao Q, Yuan B, Chess A (2000) Convergent projections of Drosophila olfactory neurons to specific glomeruli in the antennal lobe. Nat Neurosci 3:780-785

Goldman AL, Van der Goes van Naters W, Lessing D, Warr CG, Carlson JR (2005) Coexpression of two functional odor receptors in one neuron. Neuron 45:661-666.

Hallem EA, Ho MG, Carlson JR (2004) The molecular basis of odor coding in the Drosophila antenna. Cell 117:965-979.

Imai T, Suzuki M, Sakano H (2006) Odorant receptor-derived cAMP signals direct axonal targeting. Science 314:657-661.

Johansen T, Moens U, Holm T, Fjose A, Krauss S (1993) Zebrafish pou[c]; a divergent POU family gene ubiquitously expressed during embryogenesis. Nucleic Acids Res 21:475-483.

Komiyama T, Luo L (2006) Development of wiring specificity in the olfactory system. Curr Opin Neurobiol 16:67-73. 
Komiyama T, Carlson JR, Luo L (2004) Olfactory receptor neuron axon targeting: intrinsic transcriptional control and hierarchical interactions. Nat Neurosci 7:819-825.

Kreher SA, Kwon JY, Carlson JR (2005) The molecular basis of odor coding in the Drosophila larva. Neuron 46:445-456.

Laissue PP, Reiter C, Hiesinger PR, Halter S, Fischbach KF, Stocker RF (1999) Three-dimensional reconstruction of the antennal lobe in Drosophila melanogaster. J Comp Neurol 405:543-552.

Lanjuin A, VanHoven MK, Bargmann CI, Thompson JK, Sengupta P (2003) Otx/otd homeobox genes specify distinct sensory neuron identities in $C$. elegans. Dev Cell 5:621-633.

Lee T, Luo L (1999) Mosaic analysis with a repressible cell marker for studies of gene function in neuronal morphogenesis. Neuron 22:451-461.

McKenna M, Monte P, Helfand SL, Woodard C, Carlson J (1989) A simple chemosensory response in Drosophila and the isolation of acj mutants in which it is affected. Proc Natl Acad Sci U S A 86:8118-8122.

Melkman T, Sengupta P (2005) Regulation of chemosensory and GABAergic motor neuron development by the C. elegans Aristaless/Arx homolog alr-1. Development 132:1935-1949.

Mombaerts P, Wang F, Dulac C, Chao SK, Nemes A, Mendelsohn M, Edmondson J, Axel R (1996) Visualizing an olfactory sensory map. Cell 87:675-686.

Okamoto K, Wakamiya M, Noji S, Koyama E, Taniguchi S, Takemura R, Copeland NG, Gilbert DJ, Jenkins NA, Muramatsu M (1993) A novel class of murine POU gene predominantly expressed in central nervous system. J Biol Chem 268:7449-7457.

Parks AL, Cook KR, Belvin M, Dompe NA, Fawcett R, Huppert K, Tan LR, Winter CG, Bogart KP, Deal JE, Deal-Herr ME, Grant D, Marcinko M, Miyazaki WY, Robertson S, Shaw KJ, Tabios M, Vysotskaia V, Zhao L, Andrade RS, et al. (2004) Systematic generation of high-resolution deletion coverage of the Drosophila melanogaster genome. Nat Genet 36:288-292.

Ray A, van der Goes van Naters W, Shiraiwa T, Carlson JR (2007) Mechanisms of odor receptor gene choice in Drosophila. Neuron 53:353-369.

Ray A, van der Goes van Naters W, Carlson JR (2008) A regulatory code for neuron-specific odor receptor expression. PLoS Biol 6:1069-1083.

Robertson HM, Warr CG, Carlson JR (2003) Molecular evolution of the insect chemoreceptor gene superfamily in Drosophila melanogaster. Proc Natl Acad Sci U S A 100:14537-14542.

Ryan AK, Rosenfeld MG (1997) POU domain family values: flexibility, partnerships, and developmental codes. Genes Dev 11:1207-1225.

Sagasti A, Hobert O, Troemel ER, Ruvkun G, Bargmann CI (1999) Alterna- tive olfactory neuron fates are specified by the LIM homeobox gene lim-4. Genes Dev 13:1794-1806.

Sarafi-Reinach TR, Melkman T, Hobert O, Sengupta P (2001) The lin-11 LIM homeobox gene specifies olfactory and chemosensory neuron fates in C. elegans. Development 128:3269-3281.

Sengupta P, Colbert HA, Bargmann CI (1994) The C. elegans gene odr-7 encodes an olfactory-specific member of the nuclear receptor superfamily. Cell 79:971-980.

Serizawa S, Miyamichi K, Takeuchi H, Yamagishi Y, Suzuki M, Sakano H (2006) A neuronal identity code for the odorant receptor-specific and activity-dependent axon sorting. Cell 127:1057-1069.

Thibault ST, Singer MA, Miyazaki WY, Milash B, Dompe NA, Singh CM, Buchholz R, Demsky M, Fawcett R, Francis-Lang HL, Ryner L, Cheung LM, Chong A, Erickson C, Fisher WW, Greer K, Hartouni SR, Howie E, Jakkula L, Joo D, et al. (2004) A complementary transposon tool kit for Drosophila melanogaster using P and piggyBac. Nat Genet 36:283-287.

Thorne N, Chromey C, Bray S, Amrein H (2004) Taste perception and coding in Drosophila. Curr Biol 14:1065-1079.

Voss JW, Wilson L, Rosenfeld MG (1991) POU-domain proteins Pit-1 and Oct-1 interact to form a heteromeric complex and can cooperate to induce expression of the prolactin promoter. Genes Dev 5:1309-1320.

Vosshall LB, Amrein H, Morozov PS, Rzhetsky A, Axel R (1999) A spatial map of olfactory receptor expression in the Drosophila antenna. Cell 96:725-736.

Vosshall LB, Wong AM, Axel R (2000) An olfactory sensory map in the fly brain. Cell 102:147-159.

Wang F, Nemes A, Mendelsohn M, Axel R (1998) Odorant receptors govern the formation of a precise topographic map. Cell 93:47-60.

Wang JW, Wong AM, Flores J, Vosshall LB, Axel R (2003) Two-photon calcium imaging reveals an odor-evoked map of activity in the fly brain. Cell 112:271-282.

Wey E, Lyons GE, Schäfer BW (1994) A human POU domain gene, mPOU, is expressed in developing brain and specific adult tissues. Eur J Biochem 220:753-762.

Wu JS, Luo L (2006) A protocol for dissecting Drosophila melanogaster brains for live imaging or immunostaining. Nat Protoc 1:2110-2115.

Zhao H, Reed RR (2001) X inactivation of the OCNC1 channel gene reveals a role for activity-dependent competition in the olfactory system. Cell 104:651-660.

Zhou H, Yoshioka T, Nathans J (1996) Retina-derived POU-domain factor-1: a complex POU-domain gene implicated in the development of retinal ganglion and amacrine cells. J Neurosci 16:2261-2274. 